\title{
Materialentfernung in der kraniomaxillofazialen Traumatologie
}

\author{
Tobias Ebker, Christian Lohse, Henning Hanken, Max Heiland, Alexander Gröbe
}

\section{Zusammenfassung}

Frakturen des Gesichtsschädels sind eine sehr häufige Verletzungsart. Gewaltdelikte zählen neben Stürzen, Sportverletzungen und Verkehrsunfällen zu den führenden Ursachen. Die Therapie der Wahl ist heute in den meisten Fällen die Reposition und Osteosynthese mittels Miniplatten aus Titan. Das Management von eingebrachtem Osteosynthesematerial (OSM) im Gesichtsbereich wird häufig durch die Hauspolitik der jeweiligen Klinik bestimmt. Dieser Übersichtsartikel beschreibt die aktuelle Datenlage zur Entfernung von in situ befindlichem OSM in der kraniomaxillofazialen Chirurgie.

\section{Management of Hardware in Craniomaxillofacial Trauma}

Fractures of the facial skeleton are a very common health problem. Falls, sport and traffic injuries and assaults are among the leading causes of facial trauma. Facial fractures are treated by open reduction and internal fixation using titanium miniplates. This review article describes the current literature on the management of hardware in craniomaxillofacial trauma.

\section{Einleitung}

Frakturen der Gesichtsknochen gehören zu den häufigsten Frakturmustern überhaupt [1-2]. Knapp die Hälfte der traumatologischen Schockraumpatienten weisen Frakturen des Gesichtsschädels auf [3]. In der kraniomaxillofazialen Traumatologie wird zwischen Frakturen des Unterkiefers und der Mittelgesichtsknochen unterschieden. Frakturen des Mittelgesichts werden nach ihrer Lokalisation in zentrale, zentrolaterale und laterale Mittelgesichtsfrakturen unterteilt. Unterkieferfrakturen werden in Brüche des Unterkieferkörpers, des aufsteigenden Unterkieferastes, des Unterkieferhalses und des gelenktragenden Unterkieferköpfchens klassifiziert. Die häufigsten Ursachen von Gesichtsschädelfrakturen sind Gewaltdelikte, Stürze, Verkehrsunfälle und Sportverletzungen [4-5]. Die typische Patientenklientel ist männlich und im jungen Erwachsenen-

OP-JOURNAL 2016; 32: 114-118

(C) Georg Thieme Verlag KG Stuttgart · New York DOI http://dx.doi.org/10.1055/s-0042-107037 alter [6]. Unterkieferfrakturen machen dabei ca. die Hälfte der Gesichtsschädelfrakturen aus $[6,7]$ gefolgt von isolierten Frakturen des zygomatikoorbitalen Komplexes (ca. 35\%) und komplexen Mittelgesichtsfrakturen (ca. 10\%) [6]. Eine Behandlungsindikation besteht bei entsprechendem Dislokationsgrad und klinischer Symptomatik [8]. Die operative Therapie der Wahl ist heute die Osteosynthese durch Open Reduction Internal Fixation (ORIF) [9].

Die ORIF hat im Allgemeinen ein deutlich besseres funktionelles Langzeitergebnis als die nicht-operative intermaxilläre Fixation [10].

Titan ist aufgrund seiner guten Biokompatibilität [11] in der Gesichtstraumatologie das am weitesten verbreitete Osteosynthesematerial (OSM) [12]. Miniplatten aus Titan und entsprechende Schrauben aus einer härteren Titan-Aluminium-Vanadium-Legierung (Ti-6Al$4 \mathrm{~V}$ ) werden in der Osteosynthese von Gesichtsfrakturen seit Jahrzehnten erfolgreich eingesetzt (Abb. 1) [12,13]. Es gibt jedoch keine allgemeine Empfeh- lung zum postoperativen Management von eingebrachtem OSM. Dieser Artikel soll einen Überblick über die aktuelle Datenlage zur Entfernung von in situ befindlichem OSM in der kraniomaxillofazialen Chirurgie geben.

\section{Indikationen für die Entfernung von Osteosynthesematerial}

Grundsätzlich muss zwischen routinemäßiger und symptomatischer Materialentfernung (ME) unterschieden werden. In der Literatur werden Entfernungsraten entweder auf das Gesamtpatientenkollektiv (per patient) oder auf die Gesamtzahl eingebrachter Osteosyntheseplatten (per plate) bezogen. Die routinemäßige ME wird nach einem definierten Zeitintervall (ca. 6-12 Monate) ohne Vorliegen klinischer Symptome durchgeführt. Dieses Vorgehen wurde in der Mund-Kiefer-Gesichts-Chirurgie (MKGChirurgie) in der Anfangszeit der Plattenosteosynthese häufig praktiziert $[10,14]$, wird aber heute nur noch von sehr wenigen Mund-, Kiefer-, Gesichtschirurgen favorisiert [15-16]. Eine Ausnahme bilden Kieferwinkelfrakturen, bei denen Weisheitszähne im Frakturspalt erhalten wurden. In diesen Fällen wird häufig eine routinemäßige ME zur Infektionsprophylaxe vorgenommen [6].

Die symptomatische ME wird bei subjektiven oder objektiven Indikationen vorgenommen [6]. Zu den subjektiven Gründen zählen Schmerzen, Missempfindungen und eine Tastbarkeit der Osteosyntheseplatte $[12,17]$. Einige Patienten klagen über thermale Dysästhesie im Bereich der operierten Körperregion [6]. Die Genese dieser Kälteempfindlichkeit ist nicht sicher geklärt. Als pathogenetische Faktoren werden neben der eingebrachten Osteosyntheseplatte eine traumatische Nervenläsion durch den initialen Unfall oder eine Nervenschädigung im Rahmen der operativen Frakturversorgung diskutiert [18]. 


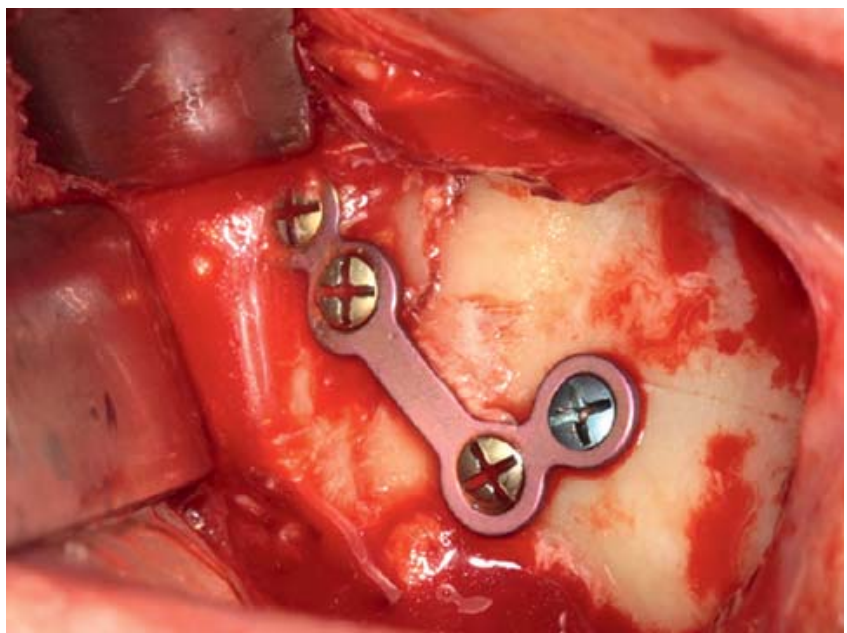

Abb. 1 Eingebrachtes OSM im Bereich des rechten Oberkiefers. Eine Mittelgesichtsfraktur im Bereich der rechten LeFort-I-Ebene wurde mit einer Miniplatte (Synthes Matrix Midface) aus Titan versorgt.
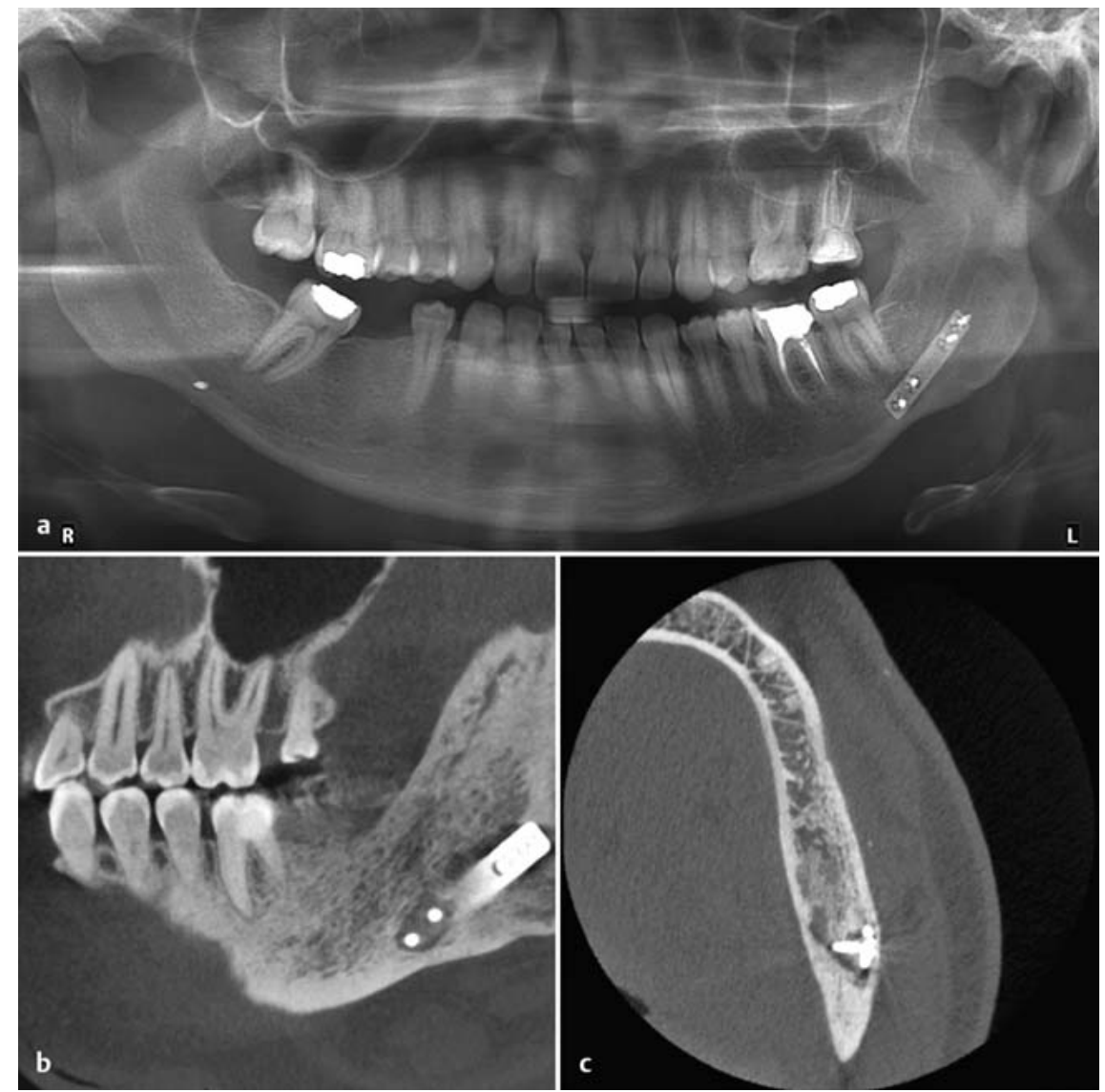

Abb. 2 a bis c Radiologische Darstellung von infiziertem OSM im Bereich des linken Unterkiefers: 4 Jahre nach osteosynthetischer Versorgung einer Unterkieferfraktur entwickelte dieser Patient eine Infektion des eingebrachten OSM. Die DVT-Aufnahmen zeigen eine Osteolyse im anterioren Bereich der inzwischen vollständig ummauerten Metallplatte (Pfeil).

Unter den objektiven Indikationen werden Plattenlockerung, Plattenfraktur [7], asymptomatische Freilegung und Infektionen (Abb.2) im Zusammenhang mit dem eingebrachten Fremdmaterial subsummiert. In situ befindliches OSM stellt einen alloplastischen Fremdkörper dar und kann trotz der hohen Biokompatibilität von Titan potenziell zu Fremdkörperreaktionen und bakterieller Besied- lung führen [12]. Eine Infektion des OSM ist die häufigste Komplikation [7]. Klinische Manifestationen sind lokale Überwärmung, Rötung, Druckschmerzhaftigkeit und putride Sekretion. Eine Platteninfektion kann zu Materialfreilegung, Fistelbildung, Pseudarthrosenbildung und schlimmstenfalls zu Osteomyelitis führen [17]. Allgemeinreaktionen wie Fieber und Abgeschlagenheit mit laborchemischen Entzündungszeichen können auftreten [17].

Die Komplikationsrate hängt dabei von der Art der Osteosynthese (mit/ohne Kompression), der Lokalisation [12], der Plattendicke [12], der Schraubenart und der Anzahl der Miniplatten ab [19]. Verglichen mit der orthopädischen Traumatologie sind für MKG-chirurgische Traumapatienten deutlich niedrigere Inzidenzraten von OSM-Infektionen beschrieben [17]. Ein entscheidender Faktor könnte die privilegierte Gewebeperfusion der Kopf-Hals-Region sein.

Komplikationen bei einliegendem OSM ereignen sich meist in den ersten 12 Monaten nach operativer Frakturversorgung [6,12-13]. Thorén et al. berichteten 2010 in einer retrospektiven Studie mit 238 Patienten, dass eine ME durchschnittlich nach ca. 9 Monaten durchgeführt wurde. $38 \%$ der Materialentfernungen wurden in den ersten 6 Monaten nach operativer Versorgung durchgeführt, $35 \%$ zwischen dem 6 . und 12 . Monat und 25\% im 2. Jahr nach Frakturversorgung. Nur $2 \%$ der Materialentfernungen waren nach einem Intervall von mehr als 2 Jahren indiziert [6]. Ist eine routinemäßige ME geplant, sollte diese in einem Zeitraum zwischen 6 und 12 Monaten nach Frakturversorgung erfolgen [20-22]. Danach steigt das Operationsrisiko durch zunehmende knöcherne Ummauerung des OSM [23].

\section{Inzidenz und Indikationen der Materialentfernung}

Die Häufigkeit einer symptomatischen ME wird in der Literatur in einem Bereich von 3,7-33,3\% angegeben $[5,6,24-$ $26,27]$. Die unterschiedliche Inzidenz ist u.a. durch inhomogene Einschlusskriterien hinsichtlich Frakturtyp und Verletzungsschweregrad erklärbar [15].Zudem ist die Materialentfernungsrate abhängig von der Gesichtsregion.

Unterkieferfrakturen sind häufiger mit Komplikationen behaftet als Mittelgesichtsfrakturen.

Neben biomechanischen Aspekten (hohe Kraftspitzen beim Kauvorgang) wird von einigen Autoren eine höhere Infektionsanfälligkeit als ursächlich angesehen [7]. Daher soll im Folgenden eine separate Betrachtung der Gesichtsregionen erfolgen. 
Materialentfernung bei Gesichtsschädelfrakturen (Gesamtkollektiv)

Hanson et al. [7] haben 2007 Daten des Nationwide Inpatient Sample (NIS), einer landesweiten US-amerikanischen Gesundheitsstudie, veröffentlicht. In dieser retrospektiven Studie wurden 4879 traumatologische Patienten erfasst, bei denen eine ORIF von Gesichtsknochen durchgeführt worden war. Bei 5\% der Patienten musste eine ME aufgrund von Komplikationen durchgeführt werden. Die 4 häufigsten Indikationen für eine ME waren Infektionen (29,4\%), Materialbrüche (18,2\%), Okklusionsstörungen (13,6\%) und Pseudarthrosenbildung (8,2\%). Nicht abgeschlossenes Körperwachstum war ein weiterer Grund für eine ME [26].

Anderen Autoren gehen von deutlich höheren Materialentfernungsraten aus. In einer retrospektiven finnischen Studie [6] mit 238 Patienten war eine ME bei 20,2\% der Patienten mit Gesichtsschädelfrakturen nötig. Explizit prophylaktische Motive lagen bei keinem Patienten vor. Objektive Indikationen führten bei $7 \%$ aller Patienten zu einer ME. In 6\% aller Fälle waren subjektive Gründe wie Kälteempfindlichkeit, Unbehagen und Palpabilität der Grund für die Plattenentfernung. Der Anteil subjektiver Indikationen für eine ME nahm von kranial (78,5\% bei zygomatikoorbitalen Frakturen) nach kaudal $(6,7 \%$ bei Manidibulafrakturen) ab $[6,24]$. Eine dünne Weichteildecke und klimatische Einflüsse scheinen bei den subjektiven Gründen für eine $\mathrm{ME}$ eine wichtige Rolle zu spielen [6,24]. In kalten Regionen wie Finnland [6] oder Kanada [24] sind diese Beschwerden häufiger anzutreffen als in wärmeren Ländern wie Griechenland [23].

Subjektive Missempfindungen treten bevorzugt auf bei OSM im Bereich der Nervenaustrittspunkte am Supra- und Infraorbitalrand, Platten im Bereich des Foramen mentale sowie in Arealen mit einer dünnen Weichteildecke und in topografischer Nähe zum M. temporalis wie der Sutura frontozygomatica [24].

\section{Materialentfernung bei Mittelgesichtsfrakturen}

In einer retrospektiven Studie von Llandro et al. [13] wurden 216 Patienten mit osteosynthetisch versorgten Mittelgesichtsfrakturen über einen Zeitraum von 6 Jahren (02/2007 bis 12/2013) untersucht. Bei $2,8 \%$ der Patienten $(n=6)$ musste das Osteosynthesematerial ent- fernt werden. 88\% des entfernten OSM war an den Stützpfeilern angebracht. Die Gründe für die symptomatische ME waren in 50\% der Fälle eine Infektion in Zusammenhang mit dem OSM und in 50\% freiliegendes OSM. 88\% des OSM musste im 1. Jahr nach Osteosynthese entfernt werden. Alle Materialentfernungen verliefen komplikationslos und in allen Fällen waren die Frakturareale konsolidiert.

\section{Materialentfernung bei Unterkieferfrakturen}

Unterkieferfrakturen sind mit einer höheren Komplikationsrate behaftet als Mittelgesichtsfrakturen [5,6,26]. Murthy et al. [5] veröffentlichten 2005 eine retrospektive Untersuchung von 76 Patientenfällen, bei denen Gesichtsschädelfrakturen osteosynthetisch versorgt wurden. Von 163 Miniplatten mussten 3,7\% (6 Platten) entfernt werden. Alle Plattenentfernungen waren bei versorgten Unterkieferfrakturen aufgrund vorliegender Infektionen nötig, obwohl an der Mandibula nur 13,5\% der Gesamtzahl der Platten $(n=22)$ angebracht waren. Rallis et al. unterstützen diese Daten [23]. In einer prospektiven Studie mit 280 Patienten musste $13,6 \%$ des OSM an der Linea obliqua und $13,2 \%$ des OSM am Unterkieferkorpus aufgrund von Komplikationen oder Patientenwunsch entfernt werden [23]. Diese Daten werden durch eine retrospektive Studie von O'Connell et al. an einem irischen Patientenkollektiv bestätigt [21]. Die Autoren haben die Daten von 434 Traumapatienten ausgewertet, die mit 800 Osteosyntheseplatten versorgt wurden. Die relativ höchste Rate einer ME lag bei ostheosythetisch versorgten Unterkieferfrakturen vor. 6,4\% der Platten (26/402) mussten bei Mandibulafrakturen entfernt werden, von denen $64 \%$ Kieferwinkelfrakturen stabilisierten. Dagegen mussten nur 2\% des OSM an der Maxilla (2/92) und 1\% der Platten (3/306) am Orbita-JochbeinKomplex entfernt werden.

Bakterielle Infektionen waren mit $42 \%$ der häufigste Grund für eine ME im Bereich der Mandibula, gefolgt von symptomatischen Weisheitszähnen (27\%) im ehemaligen Frakturareal und Plattenfreilegung (8\%).

Die Autoren postulieren, dass die relativ hohe Komplikationsrate bei Kieferwinkelfrakturen durch die dort wirkenden Kaukraftspitzen bedingt ist. Andere Autoren sehen besonders OSM im Bereich des Unterkieferkorpus als Risikofaktor für eine symptomatische ME. Mosbach et al. beschrieben in einer retrospektiven Studie an 484 Patienten mit Gesichtsschädelfrakturen eine ca. 20\%ige Entfernungsrate von OSM bei Unterkieferfrakturen verglichen mit $15 \%$ bei Oberkieferfrakturen und 2,5\% bei Jochbeinfrakturen [28]. Von der Gesamtzahl der Materialentfernungen nach Unterkieferfrakturen waren $56 \%$ bei Frakturen im Bereich des Unterkieferkörpers notwendig, 31\% im Bereich des Kieferwinkels und nur 6\% an der Symphyse. Die Hauptindikationen für die ME waren auch hier Infektionen und Wunddehiszenzen.

Zudem scheint der operative Zugang bei der Versorgung von Unterkieferfrakturen eine Rolle zu spielen. Verglichen mit einem intraoralen Zugang wiesen $\mathrm{Pa}$ tienten, die über einen extraoralen $\mathrm{Zu}$ gang versorgt wurden, eine signifikant niedrigere Rate symptomatischer Materialentfernungen auf [6].

\section{Management von infiziertem Osteosynthesematerial}

Cahill et al. haben in einem systematischen Review das Management von infiziertem Osteosynthesematerial untersucht [17]. Diese Übersichtsarbeit zeigt, dass bei infiziertem OSM unterschiedliche Behandlungsstrategien existieren. In 50\% der Publikationen wurde das OSM vollständig entfernt und in 33\% belassen, $17 \%$ der Autoren machten keine Aussage über die Art der Therapie. Diese Metaanalyse ergab keinen signifikanten Vorteil der Entfernung infizierten Osteosynthesematerials gegenüber der konservativen Therapie mittels antiseptischer Spülung und antibiotischer Therapie [17]. Die Autoren empfehlen, das Management von infiziertem OSM abhängig von der Bruchspaltkonsolidierung durchzuführen. Bei suffizienter knöcherner Durchbauung des Frakturareals sollte freiliegendes OSM entfernt werden. Liegt keine ausreichende Konsolidierung vor und stellt sich der Infekt beherrschbar dar, empfehlen die Autoren eine operative Revision mittels Inzision, Drainage, Débridement und Lavage flankiert von antibiotischer Therapie.

Befinden sich mehrere Osteosyntheseplatten in situ und muss eine Platte aufgrund von aufgetretenen Komplikationen entfernt werden, wird in der Literatur empfohlen, neben dem symptomatischen auch das asymptomatische OSM zu entfernen $[12,25]$. 
Das Entfernen von OSM ist im Allgemeinen ein komplikationsarmer operativer Eingriff, der mit geringem Flurschaden einhergeht und meist zu einem Rückgang der Beschwerden führt [12].

\section{Stress Shielding}

Das biomechanische Konzept des Stress Shielding beschreibt eine Schwächung des ortsständigen Knochens in Form einer „Inaktivitätsosteoporose“ durch das eingebrachte Osteosynthesematerial.

Stress Shielding tritt auf, wenn das Osteosynthesematerial ein deutlich höheres Elastizitätsmodul als der Knochen besitzt. Dadurch wird der Knochen vor Stress durch eingeleitete Kaukräfte „geschützt“ [29]. Dies ist v. a. bei pädiatrischen Patienten und bei Verwendung lasttragender bikortikaler Rekonstruktionsplatten problematisch $[9,30]$. Für die Bruchspaltkonsolidierung und das subsequente physiologische Remodeling des Knochens sind Mikrobewegungen im Frakturareal elementar [31]. Dieser biomechanische Stimulus verringert sich mit zunehmendem Rigiditätsgrad der Osteosynthese. Die Folge kann eine lokale Osteoporose sein [32]. Daher sollte bei kindlichem Knochen [33] und bei in situ befindlichen Rekonstruktionsplatten [34] eine routinemäßige ME vorgenommen werden. Stress Shielding tritt auch bei Miniplattenosteosynthese auf. Die Langzeiteffekte auf die Knochenstabiliät sind derzeit jedoch noch nicht ausreichend geklärt [35].

\section{Frakturen im Kindesalter}

Einen Sonderfall stellt das Management von in situ befindlichem OSM bei Kindern dar. Aufgrund des hohen Regenerations- und Remodellierungspotenzials von kindlichem Knochen wird bei entsprechender Indikation eine konservative Frakturbehandlung favorisiert [36].

Die Entwicklung von resorbierbarem OSM hat zudem die Möglichkeiten der operativen Frakturversorgung erweitert und findet zunehmend Verwendung in der pädiatrischen kraniomaxillofazialen Traumatologie [33].

Zur Prophylaxe von Wachstumsstörungen und zur Verhinderung einer passiven Migration [36] des Plattenmaterials sollte nach Einbringen von metallischem OSM eine ME erfolgen, sobald die Fraktur konsolidiert ist [33]. In der Regel wird eine Entfernung nach 2-3 Monaten empfohlen. Zudem birgt OSM im kindlichen
Kiefer mit zunehmender Plattenstärke die Gefahr der lokalen Osteoporose [33]. Lange Zeit wurde davon ausgegangen, dass eine kompromittierte Knochendurchblutung im Bereich des eingebrachten OSM ursächlich für den Festigkeitsverlust des osteosynthetisierten Knochens während der Zeit der internen Fixation ist $[14,35]$. Heute wird dagegen angenommen, dass Stress Shielding durch die reduzierte Einleitung von Kaufkräften in den ortsständigen Knochen eine wichtigere Rolle in den Knochenabbauprozessen hat $[31,33,35]$.

\section{Biokompatibilität von Titan}

Titan gilt als ein OSM mit hoher Biokompatibilität. Jedoch existieren wenig Langzeitdaten über die Auswirkungen eines dauerhaften Belassens von Osteosynthesematerial. Materialpartikel können während der Insertion oder durch kontinuierliche Abrasion unter Belastung ins Gewebe gelangen $[11,37]$. In-vitro-Untersuchungen haben zytotoxische Effekte von Titan auf Fibroblasten gezeigt [38].

In den 1990er-Jahren wurden Titanpartikel in regionalen Lymphknoten von Patienten [39] und in der Lunge, Leber und Niere von Versuchstieren [40] gefunden. Histopathologische Reaktionen oder DNA-Schädigungen konnten für diese Organablagerungen jedoch nicht nachgewiesen werden [41]. Diese älteren Untersuchungen sind allerdings mit Vorsicht zu interpretieren, da Korrosion des metallischen OSM zu dieser Zeit noch ein werkstoffkundliches Problem darstellte [40]. Die modernen Osteosynthesesysteme hingegen zeigen wenig Korrosion [42], jedoch können Partikel aus fertigungsbedingten Strukturunregelmäßigkeiten ins Gewebe gelangen [42].

In einer 12 Patienten umfassenden Studie, in der Unterkieferfrakturen mit Miniplatten aus Titan versorgt wurden, fanden Katou et al. [43] nach 6-24 Monaten Titanpartikel im Gewebe. Dies war von einer chronischen Entzündungsreaktion mit Anwesenheit von Makrophagen und T-Lymphozyten begleitet. Der Grad dieser Entzündungsreaktion wurde von anderen Autoren hingegen als mild eingestuft und als gute Gewebetoleranz interpretiert [44].

In einer Publikation von Langford et al. [11] aus dem Jahr 2002 wurden die Auswirkungen von Miniplatten aus Titan auf das umliegende Gewebe in einem Nachuntersuchungszeitraum von bis zu 13
Jahren untersucht. Im Rahmen der ME bei 35 Patienten wurden Proben aus dem umliegenden Gewebe entnommen und histologisch untersucht. Die Ergebnisse zeigten eine Gewebefibrosierung und in ca. 70\% der Fälle wurden intraund extrazelluläre titanhaltige Partikel in der unmittelbaren Umgebung des OSM gefunden. Hinweise für eine chronische Entzündung im Gewebe fanden die Autoren nicht.

\section{Empfehlungen der AOCMF}

Die AWMF-Leitlinien „laterale Mittelgesichtsfrakturen“ [45] und „Orbitadefekte, Rekonstruktion“ [46] enthalten keine allgemeinen Empfehlungen zum Management von eingebrachtem Osteosynthesematerial. Die kraniomaxillofaziale Division der Arbeitsgemeinschaft für Osteosynthesefragen (AOCMF) hingegen positioniert sich in den webbasierten Operationsempfehlungen [18] zur Versorgung von Gesichtsschädelfrakturen.

Gemäß den AOCMF-Empfehlungen ist eine Materialentfernung bei versorgten Gesichtsschädelfrakturen selten notwendig und nur bei entsprechendem Patientenwunsch sowie bei Tastbarkeit, Freilegung oder Infektion des Osteosynthesematerials indiziert.

\section{Schlussfolgerungen}

Eine Entscheidung zur routinemäßigen ME hängt wesentlich von der Definition des Komplikationsbegriffs und der individuellen Risikoauffassung $\mathrm{zu}$ eingebrachtem Fremdmaterial ab [12]. Für eine Materialentfernung sprechen das potenzielle Geweberisiko durch den alloplastischen Fremdkörper und die Komplikationsrate von 4-33\%. Argumente gegen eine routinemäßige ME sind die Risiken eines 2. operativen Eingriffs sowie die finanzielle Belastung der Kostenträger.

Das Gros der Autoren empfiehlt heute keine routinemäßige Entfernung von eingebrachtem OSM, obwohl plattenassoziierte Komplikationen nach osteosynthetischer Versorgung von Gesichtsschädelfrakturen mit einer Prävalenz von ca. 5-20\% auftreten. Komplikationen manifestieren sich i.d.R. innerhalb der ersten 12 Monate, sind aber auch nach einem längeren Zeitintervall beschrieben. Dies sollte Berücksichtigung in der Patientennachsorge finden.

Einen Sonderfall stellen kindliche Frakturen und die Frakturversorgung mit 
lasttragendem OSM dar. Hier ist eine routinemäßige ME zur Prophylaxe von Folgeschäden durch Wachstumsstörungen oder Knochenverlust durch Stress Shielding indiziert.

Die höchste Rate an objektiven Komplikationen weisen Unterkieferfrakturen auf, die über einen intraoralen Zugang versorgt wurden. Die höchste Rate subjektiver Missempfindungen durch OSM ist an anatomisch prominenten Regionen des Mittelgesichts mit einer dünnen Weichgewebsdecke zu finden. Abschließend ist festzuhalten, dass das Risiko der Plattenentfernung nach gegenwärtigem Kenntnisstand größer zu sein scheint als das Risiko des Belassens von OSM [47].

\section{Literatur}

${ }^{1}$ Motamedi MH, Dadgar E, Ebrahimi A et al. Pattern of maxillofacial fractures: a 5-year analysis of 8,818 patients. J Trauma Acute Care Surg 2014; 77: 630-634

2 Burns ST, Gugala Z, Jimenez CJ et al. Epidemiology and patterns of musculoskeletal motorcycle injuries in the USA. F1000Res 2015; 4: 114

${ }^{3}$ Whitesell RT, Steenburg SD, Shen C et al. Facial fracture in the setting of whole-body ct for trauma: incidence and clinical predictors AJR Am J Roentgenol 2015; 205: W4-10

${ }^{4}$ Gosse EM, Ferguson AW, Lymburn EG et al. Blow-out fractures: patterns of ocular motility and effect of surgical repair. $\mathrm{Br} \mathrm{J}$ Oral Maxillofac Surg 2010; 48: 40-43

${ }^{5}$ Murthy AS, Lehman JA jr. Symptomatic plate removal in maxillofacial trauma: a review of 76 cases. Ann Plast Surg 2005; 55: 603-607

${ }^{6}$ Thorén H, Snäll J, Kormi E et al. Symptomatic plate removal after treatment of facial fractures. J Craniomaxillofac Surg 2010; 38: 505-510

${ }^{7}$ Hanson J, Lovald S, Cowgill I et al. National hardware removal rate associated with internal fixation of facial fractures. J Oral Maxillofac Surg 2011; 69: 1152-1158

8 Burnstine MA. Clinical recommendations for repair of isolated orbital floor fractures: an evidence-based analysis. Ophthalmology 2002; 109: 1207-1210

9 Champy M, Lodde JP Schmitt $R$ et al Mandibular osteosynthesis by miniature screwed plates via a buccal approach. J Maxillofac Surg 1978; 6: 14-2

10 Cawood JI. Small plate osteosynthesis of mandibular fractures. Br J Oral Maxillofac Surg 1985; 23: 77-91

11 Langford RJ, Frame JW. Tissue changes adjacent to titanium plates in patients. J Craniomaxillofac Surg 2002; 30: 103-107

12 Kubota Y Kuroki T, Akita S et al. Association between plate location and plate removal following facial fracture repair. J Plast Reconst Aesthet Surg 2012; 65: 372-378

${ }^{13}$ Llandro $H$, Langford $R$. Reasons for plate removal after treatment of orbitozygomatic complex fractures. J Craniomaxillofac Surg 2015; 43: 17-20

14 Alpert B, Seligson D. Removal of asymptomatic bone plates used for orthognathic surgery and facial fractures. J Oral Maxillofac Surg 1996; 54: 618-621

${ }^{15}$ Thorén H, Snäll J, Hallermann Wet al. Policy of routine titanium miniplate removal after maxillofacial trauma. J Oral Maxillofac Surg 2008; 66: 1901-1904
${ }^{16}$ Matthew IR, Frame JW. Policy of consultant oral and maxillofacial surgeons towards removal of miniplate components after jaw fracture fixation: pilot study. Br J Oral Maxillofac Surg 1999; 37: 110-112

17 Cahill TJ 3rd, Gandhi R, Allori AC et al. Hardware removal in craniomaxillofacial trauma: a systematic review of the literature and management algorithm. Ann Plast Surg 2015; 75: 572-578

18 AO Foundation. AO Surgery Reference. Im Internet: https://www2.aofoundation.org/ wps/portal/surgery; Stand: 14.09.2015

19 Regev E, Shiff JS, Kiss A et al. Internal fixation of mandibular angle fractures: a meta-analysis. Plast Reconstr Surg 2010; 125: 17531760

20 Orringer IS, Barcelona V, Buchman SR. Reasons for removal of rigid internal fixation devices in craniofacial surgery. J Craniofac Surg 1998; 9: 40-44

21 O'Connell J, Murphy C, Ikeagwuani O et al. The fate of titanium miniplates and screws used in maxillofacial surgery: a 10 year retrospective study. Int J Oral Maxillofac Surg 2009; 38: 731-735

22 Bhatt V, Chhabra P, Dover MS. Removal of miniplates in maxillofacial surgery: a followup study. J Oral Maxillofac Surg 2005; 63: 756-760

${ }^{23}$ Rallis G, Mourouzis C, Papakosta V et al. Reasons for miniplate removal following maxillofacial trauma: a 4-year study. J Craniomaxillofac Surg 2006; 34: 435-439

${ }^{24}$ Nagase DY, Courtemanche DJ, Peters DA. Plate removal in traumatic facial fractures: 13-year practice review. Ann Plast Surg 2005; 55 : 608-611

25 Islamoglu $K$, Coskunfirat OK, Tetik $G$ et al. Complications and removal rates of miniplates and screws used for maxillofacial fractures. Ann Plast Surg 2002; 48: 265-268

${ }^{26}$ Bakathir AA, Margasahayam MV, Al-Ismaily $M I$. Removal of bone plates in patients with maxillofacial trauma: a retrospective study. Oral Surg Oral Med Oral Pathol Oral Radiol Endod 2008; 105: e32-37

27 Chaushu G, Manor Y, Shoshani Yet al. Risk factors contributing to symptomatic plate removal in maxillofacial trauma patients. Plast Reconstr Surg 2000; 105: 521-525

28 Mosbah MR, Oloyede D, Koppel DA et al. Miniplate removal in trauma and orthognathic surgery-a retrospective study. Int J Oral Maxillofac Surg 2003; 32: 148-151

${ }^{29}$ Alexander $R$, Theodos $L$. Fracture of the bonegrafted mandible secondary to stress shielding: report of a case and review of the literature. J Oral Maxillofac Surg 1993; 51: 695-697

${ }^{30}$ Kennady MC, Tucker MR, Lester GE et al. Stress shielding effect of rigid internal fixation plates on mandibular bone grafts. A photon absorption densitometry and quantitative computerized tomographic evaluation. Int J Oral Maxillofac Surg 1989; 18: 307-310

31 Uhthoff HK, Poitras P, Backman DS. Internal plate fixation of fractures: short history and recent developments. J Orthop Sci 2006; 11: 118-126

32 Patel MF, Langdon JD. Titanium mesh (TiMesh) osteosynthesis: a fast and adaptable method of semi-rigid fixation. $\mathrm{Br} J$ Oral Maxillofac Surg 1991; 29: 316-324

33 Yerit KC, Hainich S, Enislidis $G$ et al. Biodegradable fixation of mandibular fractures in children: stability and early results. Oral Surg Oral Med Oral Pathol Oral Radiol Endod 2005: 100: 17-24

34 Zoumalan RA, Hirsch DL, Levine JP et al. Plating in microvascular reconstruction of the mandible: can fixation be too rigid? J Craniofac Surg 2009; 20: 1451-1454

$35 \mathrm{Ji} \mathrm{B}$, Wang C, Liu L et al. A biomechanical analysis of titanium miniplates used for treat- ment of mandibular symphyseal fractures with the finite element method. Oral Surg Oral Med Oral Pathol Oral Radiol Endod 2010; 109: e21-e77

${ }^{36}$ Perry M. Maxillofacial trauma-developments, innovations and controversies. Injury 2009; 40: 1252-1259

${ }^{37}$ Rosenberg A, Gratz KW, Sailer HF. Should titanium miniplates be removed after bone healing is complete? Int J Oral Maxillofac Surg 1993; 22: 185-188

38 Evans EJ. Cell damage in vitro following direct contact with fine particles of titanium, titanium alloy and cobalt-chrome-molybdenum alloy. Biomaterials 1994; 15: 713-717

39 Onodera K, Ooya K, Kawamura H. Titanium lymph node pigmentation in the reconstruction plate system of a mandibular bone defect. Oral Surg Oral Med Oral Pathol 1993; 75: 495-497

40 Bessho K, Fujimura K, Iizuka T. Experimental long-term study of titanium ions eluted from pure titanium miniplates. J Biomed Mater Res 1995; 29: 901-904

41 Piozzi R, Ribeiro DA, Padovan LE et al. Genotoxicity and cytotoxicity in multiple organs induced by titanium miniplates in Wistar rats. J Biomed Mater Res A 2009; 88: 342-347

${ }^{42}$ Langford RJ, Frame JW. Surface analysis of titanium maxillofacial plates and screws retrieved from patients. Int J Oral Maxillofac Surg 2002; 31: 511-518

43 Katou F, Andoh N, Motegi K et al. Immuno-inflammatory responses in the tissue adjacent to titanium miniplates used in the treatment of mandibular fractures. J Craniomaxillofac Surg 1996; 24: 155-162

${ }^{44}$ Torgersen S, Moe G, Jonsson R. Immunocompetent cells adjacent to stainless steel and titanium miniplates and screws. Eur J Oral Sci 1995; 103: 46-54

${ }^{45}$ AWMF. Leitlinie „Laterale Mittelgesichtsfrakturen“. Im Internet: http://www.awmf.org leitlinien/detail/1l/007-016.html; Stand: 15.09.2015

46 AWMF Leitlinie Orbitadefekte, Rekonstruktion“. Im Internet: http://www.awmf.org/ leitlinien/detail/11/007-099.html; Stand: 15.09.2015

${ }^{47}$ Manor Y, Chaushu G, Taicher S. Risk factors contributing to symptomatic plate removal in orthognathic surgery patients. J Oral Maxillofac Surg 1999; 57: 679-682

\section{Dr. med. Tobias Ebker}

Wissenschaftlicher Mitarbeiter

Dr. med. Dr. med. dent.

Henning Hanken

Oberarzt

Dr. med. Christian Lohse

Wissenschaftlicher Mitarbeiter

Priv.-Doz. Dr. med. Dr. med. dent.

Alexander Gröbe

Leitender Oberarzt und

stv. Klinikdirektor

Prof. Dr. med. Dr. med. dent.

Max Heiland

Klinikdirektor

Klinik und Poliklinik für Mund-, Kiefer- und Gesichtschirurgie Universitätsklinikum HamburgEppendorf

Martinistraße 52

20246 Hamburg

a.groebe@uke.de 